\title{
Intestinal Permeability Barrier in Crohn's Disease: The Difficulty in Shifting the Paradigm
}

\author{
Daniel Hollander
}

Received: 1 April 2013/Accepted: 10 May 2013/Published online: 8 June 2013

(C) Springer Science+Business Media New York 2013

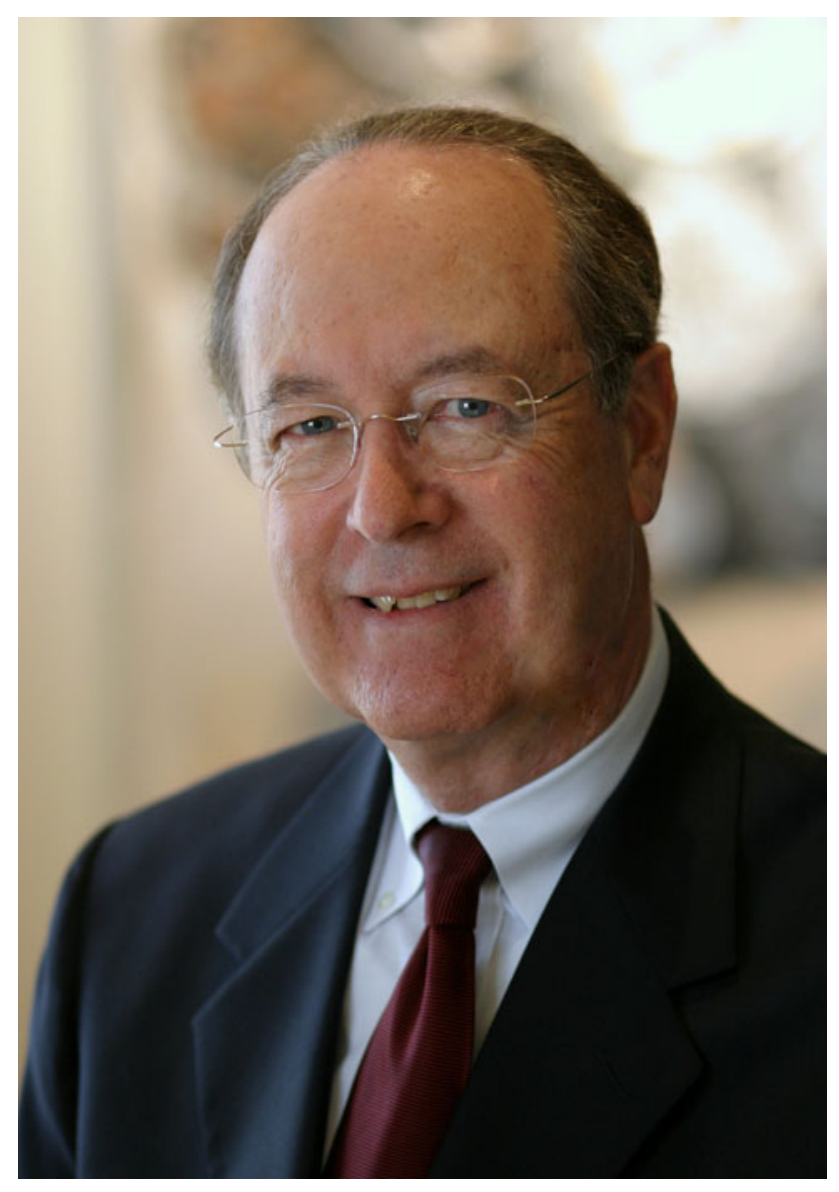

Dr. Daniel Hollander

\section{Hollander $(\bowtie)$}

Division of Digestive Diseases, UCLA School of Medicine and The Broad Foundation, 10900 Wilshire Blvd, 12th floor, Los Angeles, CA 90024, USA

e-mail: dhollander@broadmedical.org; dholland@ucla.edu
Crohn's disease has become a relatively common inflammatory disease of the intestine. The etiology of the disease is unknown despite a great deal of research by many talented investigators. During most of the twentieth century, the search for Crohn's etiology focused mostly on immunological abnormalities. This dominant paradigm hindered the approval of research proposals or manuscripts dealing with other, non-immunological etiological factors or mechanisms.

In the early 1980s my research centered on the intestinal transport of lipid compounds, in general, and fat-soluble vitamins in particular [1]. Simultaneously, I was caring for increasing numbers of patients with Crohn's disease and felt frustrated by the dearth of basic understanding of the etiology of Crohn's disease and the paucity of available therapeutic approaches. It was known that the disease had a heritable component since it had a clear familial clustering, but the specific genetic abnormalities or predisposing genetic factors were not known. Furthermore, no measurable parameters existed that would enable us to identify the disease in its subclinical phase in the healthy relatives of Crohn's disease patients.

In the early 1980s, I was approached by the father of a patient with Crohn's who was frustrated by the lack of progress in understanding the pathogenesis of the disease or its effective therapy. He searched the literature regarding Crohn's and conferred with several investigators, but he was not satisfied with the outcomes of their efforts. He asked if I would be interested in searching for new causes of Crohn's disease and stated that he was willing to fund a preliminary pilot project through his family foundation to search for new etiological leads [2].

I was aware of three small studies that looked at a newly recognized function of the intestine, namely, the barrier function of the bowel which limits the absorption of 
antigenic, infectious or toxic substances from the intestinal lumen into the systemic circulation and was referred to as "intestinal permeability." These three articles reported increased intestinal permeability in patients with active Crohn's [3-5] but did not determine whether the increase in permeability in patients with the disease was secondary to the inflammation itself or whether it was an underlying etiological factor in the pathogenesis of the disease.

Since Crohn's has a clear familial pattern and a genetic predisposition, I reasoned that measuring intestinal permeability not only in patients with the active disease but also in their healthy relatives could clarify whether the increase in intestinal permeability is an underlying etiological factor or simply a consequence of inflammation.

I was fortunate to attract Dr. Jerome Rotter, a geneticist who was studying families with Crohn's disease, to help recruit families to the study and to participate in the investigation together with his team.

We developed a simplified and subject-friendly way to measure intestinal permeability in the patients and their family members. A talented biochemist, Dr. Thomas Delahunty, joined my laboratory and developed an accurate and relatively simple method to measure permeability markers in the collected urine of the subjects [6]. We used a partially absorbed water-soluble, non-metabolized marker molecule that was ingested with a standard meal in an outpatient setting. Since the marker is not metabolized, the portion that can penetrate the intestinal permeability barrier is secreted in the urine and can be measured in a timed collection. We chose low molecular weight polyethylene glycol 400 (PEG-400) as the marker and tested its permeability and its experimental reproducibility in normal control subjects.

Once the system was ready we studied ten families with 11 members with active Crohn's and 32 of their clinically normal unaffected relatives and compared their permeability measurements with those measured in normal control subjects without a family history of Crohn's.

Over the $6 \mathrm{~h}$ of urine collection, control subjects secreted $215 \pm 26$ MG of PEG-400, while Crohn's patients secreted $514 \pm 94$ and their clinically normal relatives $566 \pm 62 \mathrm{MG}$ (Fig. 1). The twofold increase in intestinal permeability of both the patients and their clinically normal relatives indicated that the intestinal defect in the inability to exclude the permeability probes was not secondary to intestinal inflammation but rather a primary defect that could be an etiological factor in the pathogenesis of Crohn's disease. We did not think that it was the only etiological factor but, rather, a factor that could contribute to the pathogenesis of the disease by allowing antigenic, infectious or toxic substances to penetrate the intestinal barrier and to contribute to the cascade of events that culminate in active Crohn's disease.

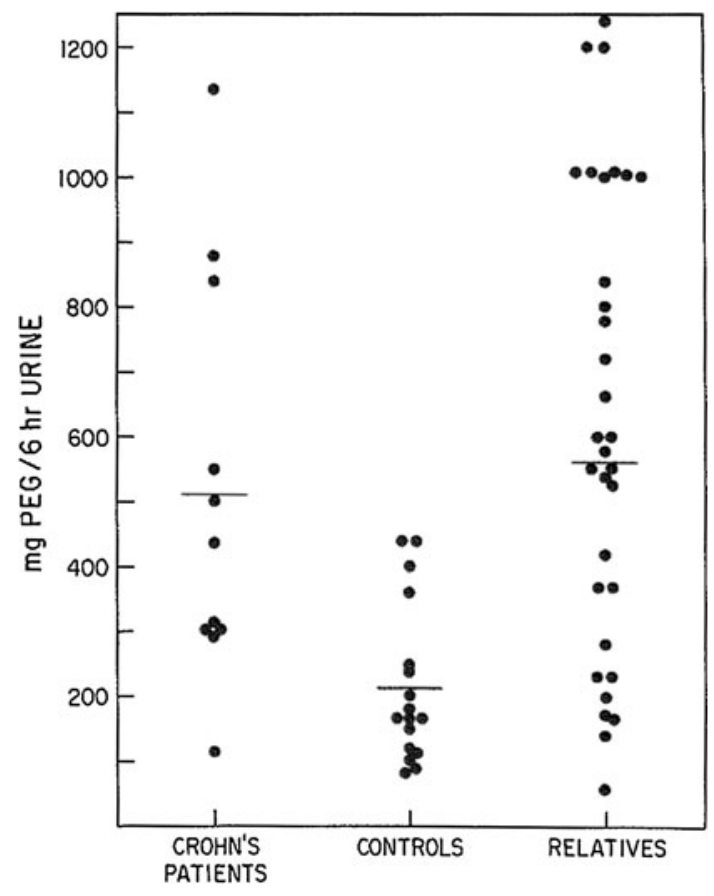

Fig. 1 Intestinal permeability in Crohn's disease patients, their healthy relatives and normal controls. Each dot represents the total PEG-400 excreted in the urine over a $6 \mathrm{~h}$ collection after the oral administration of the permeability probe. The horizontal bars are the mean value for each group. Original figure was published in ref. [7]

We submitted the article detailing the findings to several leading gastroenterology journals in succession. The article was rejected as "an idea or concept that could not have any possible bearing on Crohn's disease pathogenesis" and "had no possible research or clinical importance." After several rejections we submitted the article to a general internal medicine journal where editorial policies and reviewers were not as firmly entrenched in the dogmatic immunological paradigm of the day [7].

Now, 27 years later, abnormally increased intestinal permeability and a defective barrier in Crohn's disease is widely investigated and accepted (Fig. 2) as a useful conceptual and investigative tool for expanding our understanding of the pathogenesis of the disease. Using other permeability markers, many groups of investigators have substantiated our findings regarding a defective permeability barrier in patients with familial Crohn's disease and some of their clinically normal relatives [8, 9]. In addition, a group of investigators were able to demonstrate an increase in intestinal permeability before the onset of the Crohn's disease in a subject with increased familial risk of the disease [10]. The increase in permeability in the relatives of patients with familial Crohn's has also been shown to be accentuated in the relatives with NOD-2 mutations $[8,11]$ and to be reversible with anti-TNF alpha therapy [12]. 


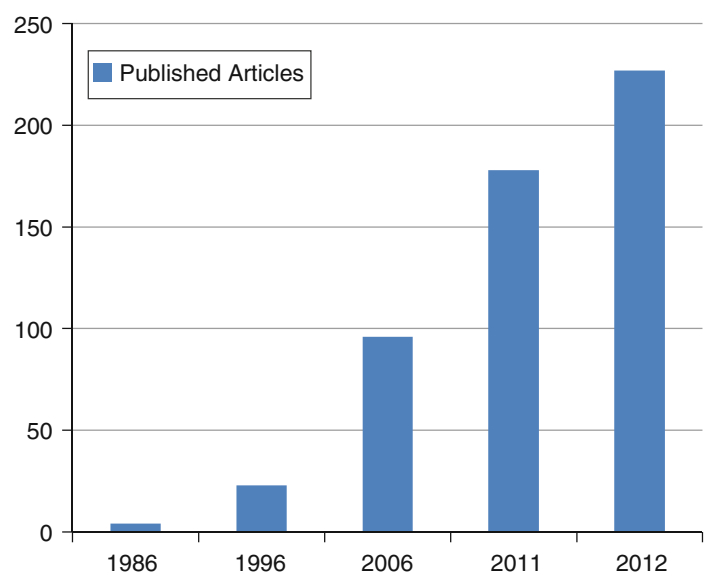

Fig. 2 The number of published articles about the "intestinal barrier" at each of the indicated years. Note: The number was obtained from the Pub Med search engine

The etiological paradigm of Crohn's disease has been expanded to include not only immunological abnormalities but also abnormalities in the structure and functions of the intestinal tight junctions [13], interactions between luminal antigens and the intestinal microbiome with the protective barrier, and immunological defenses of the intestinal mucosa. The expanded knowledge of the genetic basis of the disease provides a fuller understanding of the pathogenesis of Crohn's disease and new approaches to its therapy and prevention.

In retrospect, it was difficult to investigate and to publish a new idea that was not part of the accepted paradigm of the day. Were it not for a nontraditional source of funding for the initial clinical experiment and the willingness of patients and their family members to participate, we would not have been able to do the study. The resistance to the publication of the results was not caused by the reviewers' concerns about our approach or methodology but, rather, by their unwillingness to consider new ideas outside of the established paradigm of the time. It was disheartening to delineate data that clearly supported a new hypothesis and yet not to be able to have it accepted on the merit of the facts. However, I learned from the experience to not be leery of challenging the existing paradigm as long as the experimental design and methods are scientifically sound and as long as I was willing to anticipate the resistance of the scientific and medical community. In retrospect, it is likely that success in having the new paradigm accepted also benefitted from my having done the pivotal observations in patients with the disease rather than experiments done in cell lines or laboratory animals. Despite the initial difficulties in having a new idea accepted by the medical community, the long-term outcomes of such efforts are clearly worthwhile (Fig. 2).

Acknowledgments The author is grateful to the Goldsmith Foundation of Los Angeles for having the vision and courage to fund a new etiological investigation of Crohn's disease.

\section{References}

1. Hollander D. Intestinal absorption of vitamins A, E, D and K. $J$ Lab Clin Med. 1981;97:449-462.

2. The Goldsmith Family Foundation. Los Angeles, CA.

3. Pearson AD, Eastman EJ, Laker MF, Craft AW, Nelson R. Intestinal permeability in children with Crohn's disease and celiac disease. Br Med J. 1982;285:20-21.

4. Bjarnason I, O'Morain C, Levi AJ, Peters TJ. Absorption of 51-Chromium-labeled ethylenediaminetetracetate in inflammatory bowel disease. Gastroenterology. 1983;85:318-322.

5. Ukabam SO, Clamp JR, Cooper BT. Abnormal small intestinal permeability to sugars in patients with Crohn's disease of the terminal ileum and colon. Digestion. 1983;27:70-74.

6. Delahunty T, Hollander D. Liquid chromatographic method for estimating urinary sugars: applicability to studies of intestinal permeability. Clin Chem. 1986;32:1542-1544.

7. Hollander D, Vadheim CM, Brettholz E, Peterson GM, Delahunty TJ, Rotter JI. Increased intestinal permeability in Crohn's patients and their relatives: an etiological factor. Ann Int Med. 1986; 105:883-885.

8. Buhner S, Buning C, Genschel J, et al. Genetic basis for increased intestinal permeability in families with Crohn's disease: role of CARD 15 3020insC mutation? Gut. 2006;55:342-347.

9. Teshima CW, Dieleman LA, Meddings JB. Abnormal intestinal permeability in Crohn's disease pathogenesis. Ann N Y Acad Sci. 2012;1258:159-165.

10. Irvine EJ, Marshall JK. Increased intestinal permeability precedes the onset of Crohn's disease in a subject with familial risk. Gastroenterology. 2000;119:1740-1744.

11. D'Inca R, Annese V, di Leo V, et al. Increased intestinal permeability and NOD2 variants in familial and sporadic Crohn's disease. Aliment Pharmocol Ther. 2006;23:1455-1461.

12. Suenaert P, Bulteel V, Lemmens L, et al. Anti-tumor necrosis factor treatment restores the gut barrier in Crohn's disease. Am J Gastroenterol. 2002;97:2000-2004.

13. Suzuki T. Regulation of intestinal epithelial permeability by tight junctions. Cell Mol Life Sci. 2013;70:631-659. 